\title{
Benchmarking Monte Carlo simulations against experimental data in clinically relevant passive scattering proton therapy beamline configurations
}

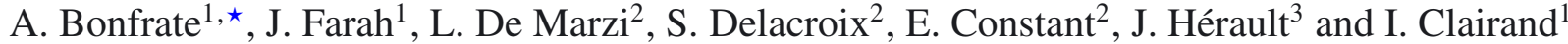 \\ ${ }^{1}$ IRSN - Laboratoire de Dosimétrie des Rayonnements Ionisants, BP17, 92262 Fontenay-aux-Roses Cedex, France. \\ 2 Institut Curie - Centre de Protonthérapie d'Orsay (CPO) - Campus universitaire bâtiment 101, 91898 Orsay, France. \\ 3 Centre Antoine Lacassagne (CAL) - Cyclotron biomédical, 227 avenue de la Lanterne, 06200 Nice, France.
}

Received 22 October 2015 - Accepted 15 February 2016

\begin{abstract}
Monte Carlo simulations are widely used to calculate secondary neutron doses in proton therapy. When using the passive scattering technique, a small variation in the tumor size or location requires a full adjustment of the beamline configuration. This work thus focuses on modeling all possible beamline elements of the local facility with the MCNPX code while covering the entire clinical range of proton beam energies. This paper documents the experimental validation of realistic beamline configurations dedicated to intracranial tumor treatments, with different sources and beamline elements. Simulations were compared against measurements and treatment planning system (TPS) data considering percentage depth dose distributions (PDD) and relative lateral dose profiles. The results show that simulated and measured PDD distributions differ by no more than $1.6 \mathrm{~mm}$ on the proton beam range (tolerance set at $2 \mathrm{~mm} / 2 \%$ ) for the $162 \mathrm{MeV}$ configuration. Meanwhile, for the modulated lateral dose profiles, the $219 \mathrm{MeV}$ configuration presented the largest difference for the in-plane field width at $90 \%$ with $2 \mathrm{~mm}$ difference in measurements. The modeled proton sources and beamline elements were validated, and the new realistic geometries can be used to calculate secondary neutron doses to healthy tissues.
\end{abstract}

Keywords: proton therapy / passive scattering / experimental validation / Monte Carlo calculations

\section{Introduction}

Since 1991, the Institut Curie - Centre de Protonthérapie d'Orsay (CPO) has used proton therapy with more than 7000 patients treated to date for ocular and intracranial tumors. Currently, the facility includes two rooms equipped with fixed beamlines as well as a room with a fully rotating gantry beamline where intracranial tumor treatments are performed using the passive double-scattering technique. This latter technique is, however, well known to involve a high level of stray neutron radiation, which could be of great concern for pediatric patients (Miralbell et al., 2002; Hall, 2006; Newhauser et al., 2009; Athar and Paganetti, 2011).

As for most external beam radiation therapy techniques, Monte Carlo (MC) calculations are increasingly used in proton therapy to assess and improve the quality of treatments. In particular, they enable the assessment of quantities often inaccessible with standard Treatment Planning Systems (TPSs) such as stray neutron doses (Mesoloras et al., 2006;

* anthony.bonfrate@irsn.fr
Fontenot et al., 2008; Zacharatou Jarlskog et al., 2008; Shin et al., 2009; Sayah et al., 2014).

However, to use MC calculations, it is first necessary to faithfully model the entire treatment room, including the nozzle with all the beamline elements as well as the room's walls, floor and ceiling. Next, a comprehensive validation is required to prove the reliability and accuracy of the MC model. Calculations are hence usually compared against experimental measurements of the proton dose, namely percentage depth dose (PDD) distributions and relative lateral dose profiles (Hérault et al., 2005; Newhauser et al., 2005; Polf et al., 2007; Sayah et al., 2013). The comparison should also be made for secondary neutron particles (Farah et al., 2014).

In the specific case of passive double-scattering proton therapy, however, the use of MC simulations requires great effort, as a small variation in the tumor size or location can impose a full adjustment or re-modeling of the entire beamline. Indeed, the tumor location and size determine the particular proton beam energy as well as the choice of beamline elements such as the two scatterers and the range-modulator wheel; together with a change in proton beam energy, a change in these 
Table 1. Additional beam line configurations validated in this study.

\begin{tabular}{lcccc}
\hline & \multicolumn{5}{c}{ Beam line configurations } \\
& $\mathbf{1}$ & Sayah et al. $\mathbf{( 2 0 1 3 )}$ & $\mathbf{2}$ & $\mathbf{3}$ \\
\hline Proton beam energy $[\mathrm{MeV}]$ & 161.96 & 178.16 & 192 & 219.69 \\
Proton beam range within water $[\mathrm{cm}]$ & 8 & 10.5 & 17 & 22 \\
\hline Modulation width $[\mathrm{cm}]$ & 7.41 & 5.6 & 7.41 & 7.41 \\
FS* & 964 & 96542 & 92 & 6432 \\
\hline RMW* & 4 & 5 & 5 & 6 \\
Number of RMW steps required & 15 & 10 & 12 & 8 \\
\hline SS* & 8 & 8 & 2 & 2 \\
Collimator aperture $[\mathrm{cm}]$ & 5.5 & 5.5 & 5.5 & 5.5 \\
\hline
\end{tabular}

* Numbers refer to the IBA terminology and not to dimensions.

three elements strongly affects secondary neutron doses. To carry out a comprehensive MC calculation of secondary neutron doses, the different dedicated beamline elements for the CPO's gantry beamline thus need to be modeled and validated.

Previous work by Sayah et al. (2013) was hence undertaken to model the CPO's gantry room and beamline in a single configuration representing one particular craniopharyngioma treatment. The current paper greatly extends the previous work by modeling all available sets of beamline elements while also covering the entire proton beam energy range regularly used at the CPO for intracranial tumor treatments, from $\sim 160 \mathrm{MeV}$ to $\sim 220 \mathrm{MeV}$ at the beamline entrance. This article hence validates three additional clinically relevant beamline configurations with different proton beam energies by comparing MC calculations against experimental measurements and TPS data in terms of PDD distributions and relative lateral dose profiles. Following this work, the methodology introduced to model the proton source as well as the entire set of beamline elements can be considered valid to simulate any clinical treatment with the CPO's gantry beamline and to calculate inherent secondary neutron doses.

\section{Materials and methods}

\subsection{Beamline configurations and validation setup}

A typical passive double-scattering proton therapy nozzle contains several beamline elements including ionization chambers, scatterers, a range-modulator wheel (RMW), jaws and a snout supporting a collimator and a compensator. Among these elements, the first scatterer (FS), the RMW and the second scatterer (SS) are the most critical elements since they enable one to shape the proton beam to the particular tumor size and location; they are hence strong sources of neutrons (Farah et al., 2014). For the CPO's gantry beamline, there are six foils available to form the FS, five different RMWs and three SSs. In clinical practice, a routine named ConvAlgo developed by the Ion Beam Applications company (IBA) is used to select these elements according to the proton beam range within the patient and the modulation width required for the treatment. The combination of the FS, RMW and SS involved in treatments was hence used in MC simulations to realistically shape the proton beam and produce stray neutron radiation.
The study by Sayah et al. (2013) focused on the modeling of the CPO's gantry room and beamline considering a $178 \mathrm{MeV}$ proton beam to treat a $5.5-\mathrm{cm}$-diameter spherical craniopharyngioma. This imposed the selection of one specific set of FS, RMW and SS ( $c f$. Table 1). The geometry and composition of these elements and the treatment room were carefully reproduced and calculations were carried out and benchmarked against experimental measurements both for protons (Sayah et al., 2013) and stray neutrons (Farah et al., 2014) to validate the MC model.

In this work, three additional beamline configurations were specifically considered to cover the entire proton beam energy range clinically used at the CPO for intracranial tumor treatments (160-220 MeV at the nozzle entrance). Table 1 documents the tested beamline configurations involving various combinations of FS foils, two new RMWs and one SS, while a fixed modulation width of $7.41 \mathrm{~cm}$ was chosen to test a significant number of RMW steps during the validation process (cf. Section 2.2). Additionally, Table 2 presents the characteristics of the different FS foils, RMWs and SSs used and shows that combinations of the FS foils and SSs mainly differ by their thickness, while RMWs can also implicate different materials. Figure 1 shows a schematic representation of the complete CPO gantry beamline together with pictures of the FS mechanism, a particular RMW and the wheel carrying the different SSs.

Finally, to simulate proton beams of different energies, three different sources were modeled according to the method established by Sayah et al. (2013). From the mean energy value given by ConvAlgo, a Gaussian distribution is selected while fixing the standard deviation to a value adapted to the proton beam used at the CPO. Then, energetic full-width at half maximum (FWHM), upper and lower energy bounds, spatial FWHM and the beam divergence are calculated. Table 3 recaps these characteristics for each source considered in this study.

\subsection{MC calculations}

In this study, the Monte Carlo N-Particle eXtended (MCNPX) transport code (version 2.6c; Pelowitz, 2008) was used to model the CPO's gantry room. This MC code has been 
Table 2. Thickness, material and density of the simulated first scatterer foils, range-modulator wheels and second scatterers. Data were provided by the IBA company.

\begin{tabular}{|c|c|c|c|c|}
\hline & $\begin{array}{l}\text { Number of } \\
\text { the element* }\end{array}$ & Thickness [mm] & Material & $\begin{array}{l}\text { Material density } \\
\qquad\left[\mathrm{g} \mathrm{cm}^{-3}\right]\end{array}$ \\
\hline \multirow{6}{*}{ FS } & 2 & 0.11 & \multirow{6}{*}{ Tantalum } & \multirow{6}{*}{16.65} \\
\hline & 3 & 0.45 & & \\
\hline & 4 & 1.11 & & \\
\hline & 5 & 0.24 & & \\
\hline & 6 & 0.03 & & \\
\hline & 9 & 1.11 & & \\
\hline \multirow{6}{*}{ RMW } & \multirow{2}{*}{4} & From 2.37 to 147.96 & Lexan & 1.2 \\
\hline & & From 0.14 to 2.44 & Lead & 11.35 \\
\hline & \multirow{2}{*}{5} & From 2.49 to 140.04 & Lexan & 1.2 \\
\hline & & From 0.12 to 3.17 & Lead & 11.35 \\
\hline & \multirow{2}{*}{6} & From 1.08 to 139.43 & Carbon & 1.84 \\
\hline & & From 1.95 to 4.25 & Lead & 11.35 \\
\hline \multirow{6}{*}{ SS } & \multirow{3}{*}{2} & Center: 0.03 & \multirow[t]{2}{*}{ Lexan } & \multirow[t]{2}{*}{1.2} \\
\hline & & Side: 29.93 & & \\
\hline & & $\begin{array}{c}\text { Center: } 6.24 \\
\text { Side: } 0.2\end{array}$ & Lead & 11.35 \\
\hline & \multirow{3}{*}{8} & Center: 37.5 & \multirow{2}{*}{ Lexan } & \multirow{2}{*}{1.2} \\
\hline & & Side: 54.04 & & \\
\hline & & $\begin{array}{c}\text { Center: } 3.46 \\
\text { Side: } 0.01\end{array}$ & Lead & 11.35 \\
\hline
\end{tabular}

* Numbers refer to the IBA terminology and not to dimensions.

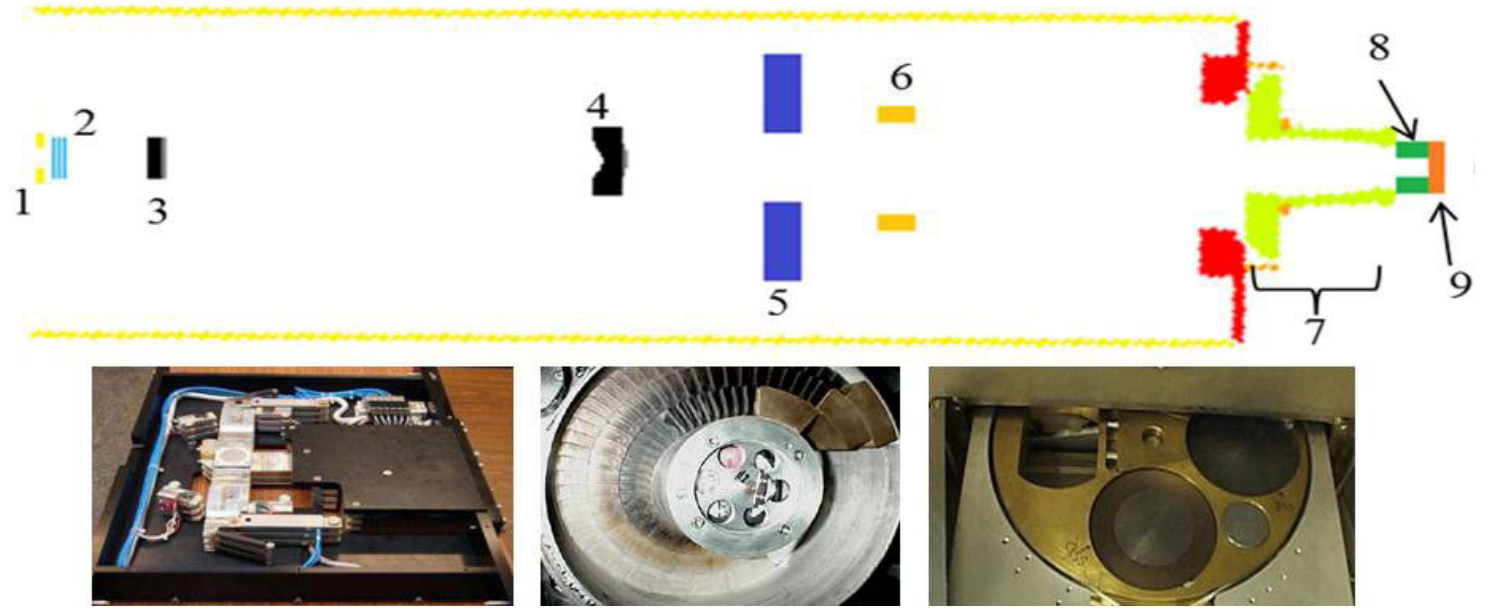

Figure 1. Schematic representation of the CPO's gantry beamline (above) together with pictures (below) of the first scatterer mechanism (left), the range-modulator wheel (middle) and the second scatterer system (right). The listed elements include: the first ionization chamber (1), the first scatterer (2), the range-modulator wheel (3), the second scatterer (4), the jaws (5), the second ionization chamber (6) and the snout (7) supporting the final collimator (8) and compensator (9).

widely used in similar proton therapy applications and extensively benchmarked against measurements (Hérault et al., 2005, 2007; Newhauser et al., 2005; Polf et al., 2007; Sayah et al., 2013; Farah et al., 2014). The modeling was done based on original engineering and technical drawings provided by the manufacturer IBA, while the exact materials and densities were assigned to the whole MCNPX geometry. Figure 2 presents the two SSs modeled with MCNPX.
To score the PDD distributions and the relative lateral dose profiles with MCNPX, a "type 3" cylindrical and rectangular mesh tally, respectively (energy deposition mesh tally) were used to calculate the energy deposition of the proton beam along its path; results are given per volume unit and per proton particle. The grid size for the PDD distributions was $1 \mathrm{~mm}$, while for the lateral dose profiles, $1 \mathrm{~mm}$ was used for the penumbra and $4 \mathrm{~mm}$ for the plateau region. To obtain 
Table 3. Characteristics of the sources considered in this study and defined in MCNPX.

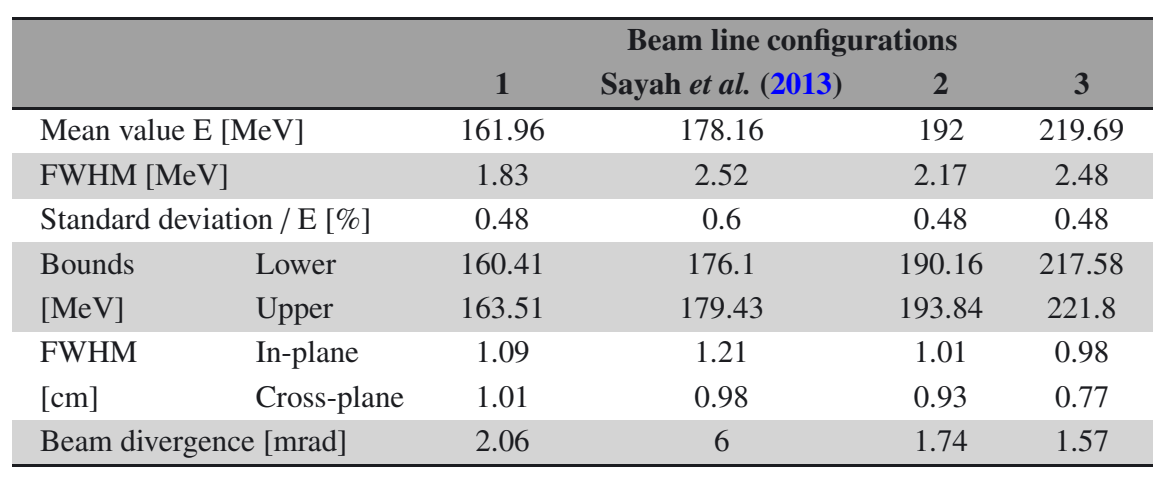

the required SOBP width, the modulation process was reproduced as described in Sayah et al. (2013), which enables the total dose calculation using weighting factors associated with each RMW step simulated. Modulated lateral dose profiles were calculated for both in-plane and cross-plane directions at two depths in the water phantom: at its entrance and at the end of the SOBP (i.e. identical to the proton beam range within water). Meanwhile, the PDD distributions were scored from the phantom entrance to a few $\mathrm{cm}$ beyond the distal penumbra.

The number of simulated protons for each pristine Bragg peak was $1 \times 10^{8}$ to ensure a statistical uncertainty within $1 \%$ in the plateau region of both the SOBP and the modulated lateral dose profiles. In addition, only protons, neutrons and photons were tracked during these simulations as per the literature (Hérault et al., 2005; Sayah et al., 2013). The LA150H proton reaction cross-section library was used (Chadwick, 1998; Chadwick et al., 1999) together with the Bertini/Dresner nuclear models (Bertini, 1963; Dresner, 1962). Physics options included Vavilov's theory for the energy straggling (Vavilov, 1957) and Rossi and Greisen's theory for the Multiple Coulomb Scattering (MCS) (Rossi and Greisen, 1941). Multiprocessing was used for each simulation to substantially decrease the simulation time; the use of 24 parallel processors of $2400 \mathrm{MHz}$ enabled an execution time of approximately 14 hours per simulation.

\subsection{Comparison criteria and validation data}

\subsubsection{Comparison criteria}

To validate MC calculations of PDD distributions, three typical distances which characterize the proton beam quality were compared: the proton beam range, the modulation width and the distal penumbra. The proton beam range is defined as the distance from the phantom entrance to the distal depth where the relative dose decreases to $90 \%$ of the maximum dose $\left(\mathrm{z}_{\mathrm{d} 90}\right)$. The modulation width corresponds to the SOBP plateau region, calculated as the distance between the proximal depth where the relative dose increases up to $95 \%\left(z_{p 95}\right)$, and the distal depth where the relative dose decreases to $95 \%\left(\mathrm{z}_{\mathrm{d} 95}\right)$. Finally, the distal penumbra represents the distance where the relative dose exhibits a falloff from $80 \%\left(\mathrm{z}_{\mathrm{d} 80}\right)$ to $20 \%\left(\mathrm{z}_{\mathrm{d} 20}\right)$ (a)

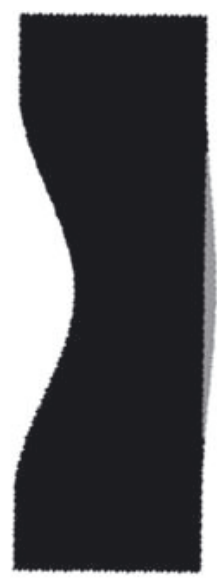

(b)

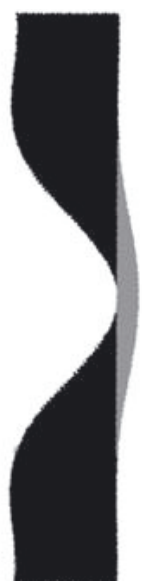

Figure 2. View of the two second scatterers considered in this study as modeled in MCNPX (transverse plane). SS 8 (a) and SS 2 (b) are represented here. The Lexan part is represented in black, while the lead part is represented in gray.

of the maximum dose. Meanwhile, to validate relative modulated lateral dose profile calculations, three additional values were calculated: both the field widths at $90 \%$ of the maximum dose $\left(\mathrm{z}_{90}\right)$ and at half maximum, i.e. at $50 \%\left(\mathrm{z}_{50}\right)$, and the lateral penumbra which represents the distance between the dose falloff from $80 \%$ to $20 \%$ of the maximum dose, namely $\mathrm{z}_{80}$ and $\mathrm{z}_{20}$, respectively. These comparison criteria are represented in Figure 3. To calculate all comparison criteria (range, FWHM, etc.), a linear interpolation was performed between the two distances which surround these criteria.

\subsubsection{Experimental measurement data}

Experimental measurements provide reference data to validate MC calculations. For this task, a water tank phantom of $65 \times 65 \times 65 \mathrm{~cm}^{3}$ was irradiated with the different beamline configurations documented in Table 1, while PDD distributions were acquired with a PPC05 plane parallel ionization chamber (IC) and relative modulated lateral dose profiles with a CC01 air compact IC from IBA (IBA, 2007). These devices have small active volumes of $0.05 \mathrm{~cm}^{3}$ and $0.01 \mathrm{~cm}^{3}$, respectively, are suitable for relative proton dose measurements in 

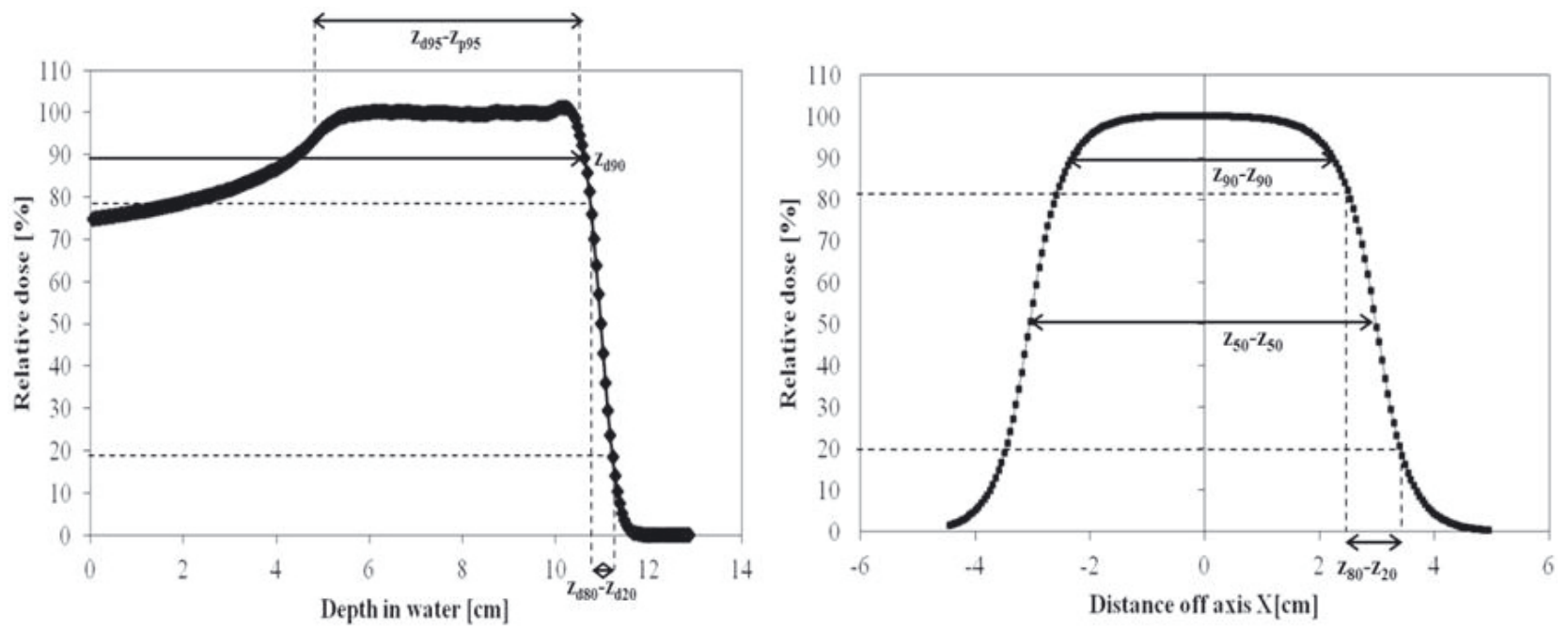

Figure 3. Comparison criteria of PDD distributions (left) and relative modulated lateral dose profiles (right) used to validate MC calculations. Index "p" refers to proximal while index "d" refers to distal.

water and have been shown to have excellent behavior for proton dose measurements (Sayah et al., 2013). Measurements with such an IC involve calibration, reading and positioning uncertainties which add up to an overall value of $3 \%$. Furthermore, the accuracy of the gantry when delivering the Bragg peaks is $\sim 1 \mathrm{~mm}$, while the IC position along the beam path is known with $0.5 \mathrm{~mm}$ uncertainty. Thus, a conservative error of $2 \mathrm{~mm}$ for the validation criterion can be considered acceptable when comparing MCNPX calculations against experimental measurements. This accuracy is sufficient considering the margins typically used in proton therapy as well as the end-use of this MC model, namely the study of secondary neutron doses to healthy tissues which involve much higher uncertainties owing to limitations on nuclear models and cross-sections.

\subsubsection{TPS data}

The TPS used in this study is ISOgray ${ }^{\circledR}$, developed in France by DOSIsoft (DOSIsoft, Cachan, France) in collaboration with the CPO. Since 2010 and after extensive commissioning and validation, this system is routinely used to plan the treatments involving the gantry beamline. Dose calculations performed with ISOgray ${ }^{\circledR}$ are done using the pencil beam algorithm (Gottschalk et al. 1993; Hong et al., 1996; Szymanowski et al., 2001) with a resolution of $1 \mathrm{~mm}$ in the three directions of space. This algorithm has shown very good agreement with experimental measurements within $1 \mathrm{~mm}$ for the lateral penumbra and for the depth dose profile (Hong et al., 1996). In addition, the algorithm applies correction for dose calculations in the presence of tissue heterogeneities. The profiles provided by the TPS can therefore be considered accurate and can be compared against $\mathrm{MC}$ calculations in the same way as measured profiles. Hence, an agreement between MC calculations and TPS data within the order of magnitude of $2 \mathrm{~mm}$ can be considered as fully satisfactory for the purpose of validating the three beamline configurations.

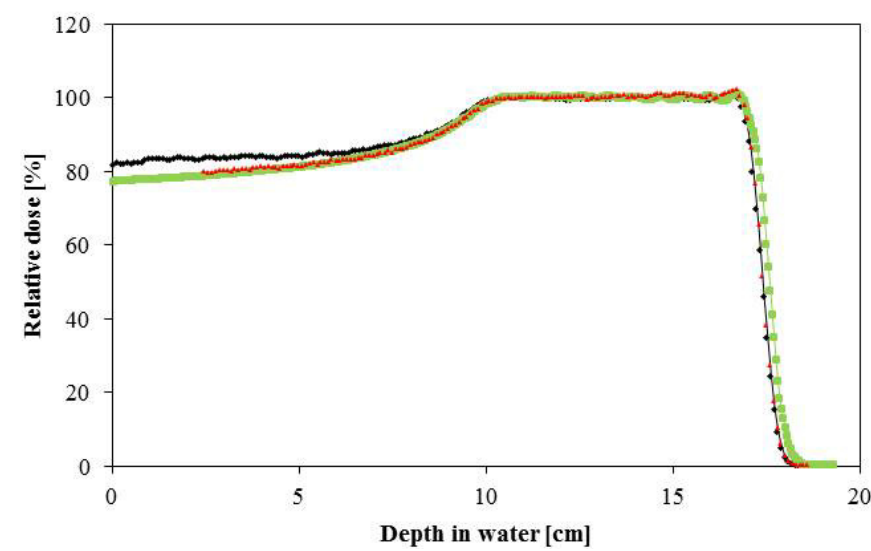

Figure 4. Comparison of PDD distributions obtained with MCNPX calculations (black diamond) against experimental measurements (red triangle) and TPS data (green square) for the $192 \mathrm{MeV}$ beamline configuration.

\section{Results}

\subsection{Validation for PDD distributions}

Figure 4 shows the PDD distributions obtained with experimental measurements, TPS data and MCNPX calculations for the $192 \mathrm{MeV}$ beamline configuration, corresponding to a proton beam range within water of $17 \mathrm{~cm}$. A slight overestimation at the beam entrance within water of $\sim 5 \%$ is observed with MC calculations; this is likely due to the proton cross-section libraries used in MCNPX code as well as the weighting factors assigned for each simulation step reproducing the RMW. This overestimation was also observed with the two other beamline configurations. Table 4 compares MC calculations against experimental measurements and TPS data for all the comparison criteria described in Section 2.3.1. The results show an overall good agreement between the simulations and experimental measurements, with a maximum difference on the proton beam range of $1.6 \mathrm{~mm}$ for the $162 \mathrm{MeV}$ and $219 \mathrm{MeV}$ 
Table 4. Comparison of MCNPX calculations against experimental measurements and TPS data in terms of proton beam range ( $\left.\mathrm{z}_{\mathrm{d} 90}\right)$, modulation width $\left(\mathrm{Z}_{\mathrm{d} 95}-\mathrm{Z}_{\mathrm{p} 95}\right)$ and distal penumbra $\left(\mathrm{Z}_{\mathrm{d} 80}-\mathrm{Z}_{\mathrm{d} 20}\right)$.

\begin{tabular}{|c|c|c|c|c|c|}
\hline $\begin{array}{c}\text { Proton } \\
\text { beam configuration }\end{array}$ & & & $\begin{array}{c}\text { Comparison values } \\
{[\mathrm{cm}]}\end{array}$ & $\begin{array}{c}\text { Measurement - MCNPX } \\
{[\mathrm{mm}]}\end{array}$ & $\begin{array}{c}\text { TPS - MCNPX } \\
{[\mathrm{mm}]}\end{array}$ \\
\hline \multirow{9}{*}{$162 \mathrm{MeV}$} & \multirow{3}{*}{$\mathrm{z}_{\mathrm{d} 90}$} & Measurements & 8.14 & \multirow{3}{*}{-1.6} & \multirow{3}{*}{-1.8} \\
\hline & & TPS & 8.12 & & \\
\hline & & MCNPX & 8.30 & & \\
\hline & \multirow{3}{*}{$\mathrm{Z}_{\mathrm{d} 95}-\mathrm{Z}_{\mathrm{p} 95}$} & Measurements & N.A. & \multirow{3}{*}{ N.A. } & \multirow{3}{*}{-0.6} \\
\hline & & TPS & 7.46 & & \\
\hline & & MCNPX & 7.52 & & \\
\hline & \multirow{3}{*}{$\mathrm{z}_{\mathrm{d} 80}-\mathrm{Z}_{\mathrm{d} 20}$} & Measurements & 0.43 & \multirow{3}{*}{0.3} & \multirow{3}{*}{0.3} \\
\hline & & TPS & 0.43 & & \\
\hline & & MCNPX & 0.40 & & \\
\hline \multirow{9}{*}{$192 \mathrm{MeV}$} & \multirow{3}{*}{$\mathrm{z}_{\mathrm{d} 90}$} & Measurements & 17.06 & \multirow{3}{*}{0.6} & \multirow{3}{*}{1.5} \\
\hline & & TPS & 17.15 & & \\
\hline & & MCNPX & 17.00 & & \\
\hline & \multirow{3}{*}{$\mathrm{Z}_{\mathrm{d} 95}-\mathrm{Z}_{\mathrm{p} 95}$} & Measurements & 7.46 & \multirow{3}{*}{-0.8} & \multirow{3}{*}{-0.6} \\
\hline & & TPS & 7.48 & & \\
\hline & & MCNPX & 7.54 & & \\
\hline & \multirow{3}{*}{$\mathrm{Z}_{\mathrm{d} 80}-\mathrm{Z}_{\mathrm{d} 20}$} & Measurements & 0.50 & \multirow{3}{*}{-0.2} & \multirow{3}{*}{-0.2} \\
\hline & & TPS & 0.50 & & \\
\hline & & MCNPX & 0.52 & & \\
\hline \multirow{9}{*}{$219 \mathrm{MeV}$} & \multirow{3}{*}{$\mathrm{z}_{\mathrm{d} 90}$} & Measurements & 22.14 & \multirow{3}{*}{-1.5} & \multirow{3}{*}{-0.2} \\
\hline & & TPS & 22.27 & & \\
\hline & & MCNPX & 22.29 & & \\
\hline & \multirow{3}{*}{$\mathrm{Z}_{\mathrm{d} 95}-\mathrm{Z}_{\mathrm{p} 95}$} & Measurements & 7.31 & \multirow{3}{*}{-1.1} & \multirow{3}{*}{0.1} \\
\hline & & TPS & 7.43 & & \\
\hline & & MCNPX & 7.42 & & \\
\hline & \multirow{3}{*}{$\mathrm{Z}_{\mathrm{d} 80}-\mathrm{Z}_{\mathrm{d} 20}$} & Measurements & 0.57 & \multirow{3}{*}{-0.4} & \multirow{3}{*}{-0.4} \\
\hline & & TPS & 0.57 & & \\
\hline & & MCNPX & 0.61 & & \\
\hline
\end{tabular}

beamline configurations. The differences between MC calculations and TPS data are also lower than $2 \mathrm{~mm}$ for the three beamline configurations and all the comparison criteria. Table 4 also shows that, in this simple water tank geometry, MC calculations are slightly outperformed by the TPS in the reproduction of proton PDD distributions. This can reasonably be explained by the simple geometry used for the validation; nonetheless, the simulation accuracy is expected to largely exceed that of TPS in more complex geometries. It should also be specified that the modulation width is not available for the $162 \mathrm{MeV}$ beamline configuration since the proximal depth of the SOBP $\left(\mathrm{z}_{\mathrm{p} 95}\right)$ was located within the wall of the water tank and was therefore technically not measurable with the IC. Finally, it is worth mentioning that the observed differences between MCNPX calculations and both experimental measurements and TPS data are expected to have little effect on the production of secondary particles. Therefore, the three beamline configurations can be considered of good accuracy when calculating secondary neutron doses to healthy tissues for radiation protection purposes.

\subsection{Validation for modulated lateral dose profiles}

Figure 5 shows the modulated lateral dose profiles, both in the in-plane and cross-plane directions, at the water phantom entrance and at the end of the SOBP for the $192 \mathrm{MeV}$ beamline configuration. Although small variations in measured proton dose values are visible at the entrance of the phantom, the field symmetry remains, however, within $1 \%$, a value that is fully acceptable for clinical treatments. Figure 5 also demonstrates that the simulations correctly reproduce the lateral dose profiles, particularly at the plateau region, while they slightly differ at the penumbra tail. Larger penumbra were indeed obtained at the end of the SOBP (Figures 5c and 5d) compared with the penumbra registered at the entrance of the water phantom (Figures 5a and 5b). This can be explained by the increasing lateral spreading of protons following nuclear reactions with increasing depth in water.

Tables 5 and 6 compare modulated lateral dose profiles obtained with MCNPX calculations, experimental measurements and the TPS at the water phantom entrance and at the end of 

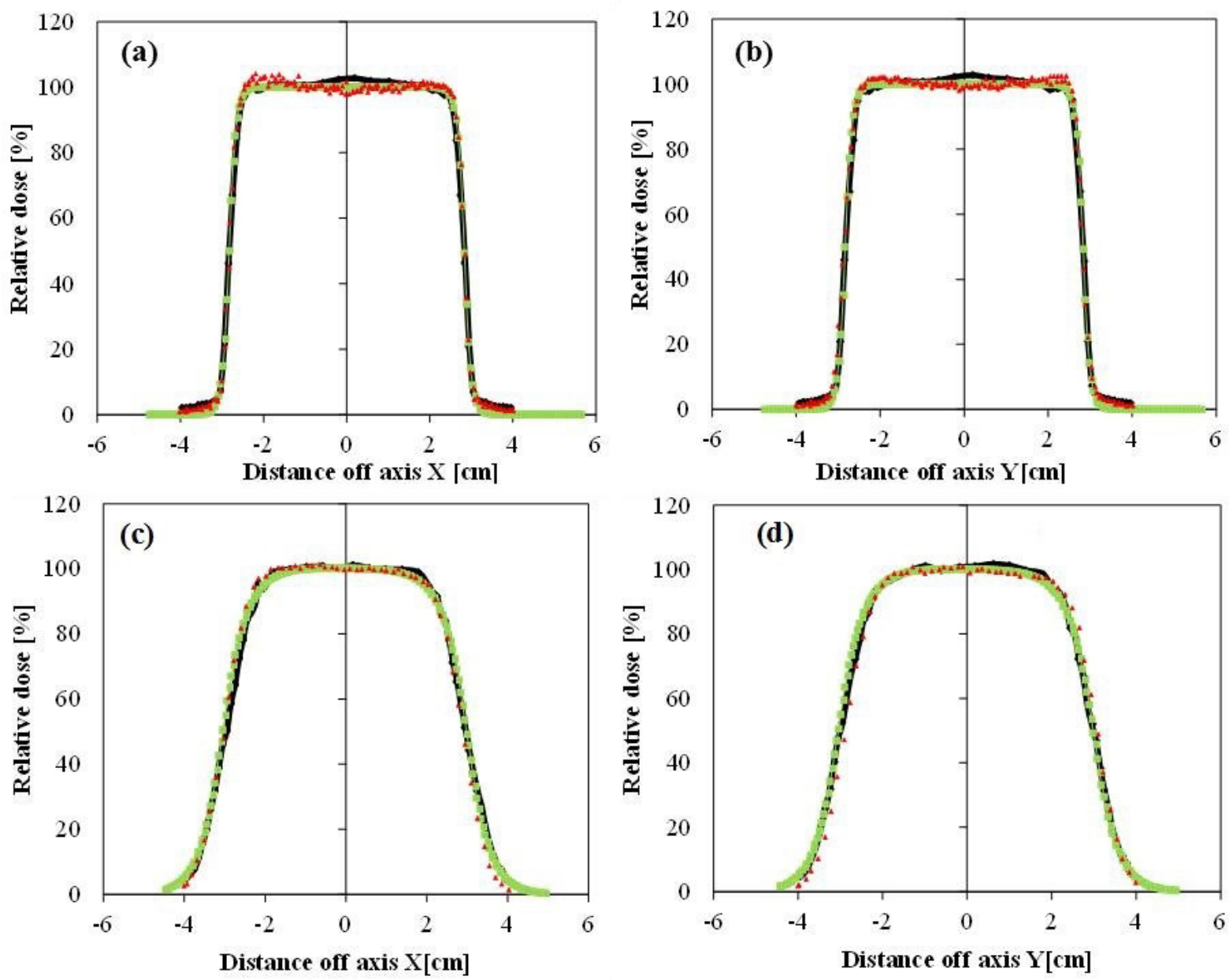

Figure 5. Comparison of modulated lateral dose profiles obtained with MCNPX calculations (black diamond) against experimental measurements (red triangle) and TPS data (green square) for the $192 \mathrm{MeV}$ beamline configuration. Data are shown for in-plane (a) and cross-plane (b) directions at the phantom entrance and for in-plane (c) and cross-plane (d) directions at the end of the SOBP (depth of $17 \mathrm{~cm}$ ).

the SOBP, respectively. At the phantom entrance, field widths at $90 \%$ and $50 \%$ as well as lateral penumbra were all within the 2 -mm validation criterion for both the in-plane and the cross-plane directions and for all the beamline configurations. At the end of the SOBP, the largest difference of $2 \mathrm{~mm}$ between MCNPX calculations and experimental measurements was observed for the field width at $90 \%$ in the in-plane direction and for the $219 \mathrm{MeV}$ beamline configuration. Again, this result is fully satisfactory considering measurement uncertainties of $\sim 3 \%$ (including the position of the IC). This proves that the proton source and all beamline element modeling was correctly done and that MCNPX calculations could be used for treatment planning in the case of complex tumor geometries.

\section{Discussion and limits}

The 2-mm validation criterion was fulfilled when comparing PDD distributions and relative lateral dose profiles obtained with MCNPX calculations against experimental measurements and TPS data for the three different beamline configurations considered in this study ( $c f$. Table 1).
The good agreement between the calculations and experimental measurements of proton beam ranges, modulation widths, field widths, and distal and lateral penumbras is comparable with values in the literature (Hérault et al., 2005; Newhauser et al., 2005; Polf et al., 2007; Sayah et al., 2013; Faddegon et al., 2015). This proves the reliability of the methodology used to determine the characteristics required in the source definition of MCNPX to correctly model the proton source of the CPO's gantry beamline. Therefore, it ensures that the validation performed in this study for the three particular proton beam energies can be extended to others. Moreover, it confirms the correct choice of nuclear models, cross-section libraries and physics options in MCNPX which enable realistic reproduction of proton interactions with matter.

However, the validation carried out in this article only studied the capacity of the MC model to reproduce the behavior of proton particles and disregarded the production of secondary radiation. Indeed, changes in beamline elements and in proton beam energy could influence the production of neutrons and the energy spectrum. Hence, a validation of the model with the new beamline configurations is needed for neutron particles 
Table 5. Comparison of MCNPX calculations against experimental measurements and TPS data in terms of field width at $90 \%$ ( $\left.\mathrm{z}_{90}-\mathrm{Z}_{90}\right)$ and half maximum $\left(\mathrm{z}_{50}-\mathrm{Z}_{50}\right)$ and lateral penumbra $\left(\mathrm{z}_{80}-\mathrm{Z}_{20}\right)$ obtained at the phantom entrance.

\begin{tabular}{|c|c|c|c|c|c|c|c|c|}
\hline \multicolumn{3}{|c|}{$\begin{array}{c}\text { Depth: } \\
\text { Phantom entrance }\end{array}$} & \multicolumn{3}{|c|}{ In-plane direction $(X)$} & \multicolumn{3}{|c|}{ Cross-plane direction (Y) } \\
\hline & & & $\begin{array}{c}\text { Comp. } \\
\text { values } \\
{[\mathrm{cm}]} \\
\end{array}$ & $\begin{array}{c}\text { Meas. - } \\
\text { MCNPX } \\
{[\mathrm{mm}]}\end{array}$ & $\begin{array}{c}\text { TPS - } \\
\text { MCNPX } \\
{[\mathrm{mm}]}\end{array}$ & $\begin{array}{c}\text { Comp. } \\
\text { values } \\
{[\mathrm{cm}]} \\
\end{array}$ & $\begin{array}{c}\text { Meas. - } \\
\text { MCNPX } \\
{[\mathrm{mm}]}\end{array}$ & $\begin{array}{c}\text { TPS - } \\
\text { MCNPX } \\
{[\mathrm{mm}]}\end{array}$ \\
\hline \multirow{9}{*}{$162 \mathrm{MeV}$ conf. } & \multirow{3}{*}{$\mathrm{Z}_{90}-\mathrm{Z}_{90}$} & Measurements & 5.20 & & & 5.18 & & \\
\hline & & TPS & 5.19 & 1.5 & 1.4 & 5.19 & 1.1 & 1.2 \\
\hline & & MCNPX & 5.05 & & & 5.07 & & \\
\hline & \multirow{3}{*}{$\mathrm{Z}_{50}-\mathrm{Z}_{50}$} & Measurements & 5.66 & & & 5.67 & & \\
\hline & & TPS & 5.66 & 0.1 & 0.1 & 5.66 & 0.2 & 0.1 \\
\hline & & MCNPX & 5.65 & & & 5.65 & & \\
\hline & \multirow{3}{*}{$\mathrm{Z}_{80}-\mathrm{Z}_{20}$} & Measurements & 0.28 & & & 0.28 & & \\
\hline & & TPS & 0.28 & -0.7 & -0.7 & 0.28 & -0.7 & -0.7 \\
\hline & & MCNPX & 0.35 & & & 0.35 & & \\
\hline \multirow{9}{*}{$192 \mathrm{MeV}$ conf. } & \multirow{3}{*}{$\mathrm{Z}_{90}-\mathrm{Z}_{90}$} & Measurements & 5.27 & & & 5.25 & & \\
\hline & & TPS & 5.27 & 0.9 & 0.9 & 5.27 & 0.6 & 0.8 \\
\hline & & MCNPX & 5.18 & & & 5.19 & & \\
\hline & \multirow{3}{*}{$\mathrm{z}_{50}-\mathrm{Z}_{50}$} & Measurements & 5.66 & & & 5.69 & & \\
\hline & & TPS & 5.66 & 0 & 0 & 5.66 & 0.3 & 0 \\
\hline & & MCNPX & 5.66 & & & 5.66 & & \\
\hline & \multirow{3}{*}{$\mathrm{Z}_{80}-\mathrm{Z}_{20}$} & Measurements & 0.23 & & & 0.26 & & \\
\hline & & TPS & 0.24 & -0.6 & -0.5 & 0.24 & -0.3 & -0.5 \\
\hline & & MCNPX & 0.29 & & & 0.29 & & \\
\hline \multirow{9}{*}{$219 \mathrm{MeV}$ conf. } & \multirow{3}{*}{$\mathrm{Z}_{90}-\mathrm{Z}_{90}$} & Measurements & 5.27 & & & 5.26 & & \\
\hline & & TPS & 5.29 & 0.9 & 1.1 & 5.29 & 0.8 & 1.1 \\
\hline & & MCNPX & 5.18 & & & 5.18 & & \\
\hline & \multirow{3}{*}{$\mathrm{z}_{50}-\mathrm{Z}_{50}$} & Measurements & 5.66 & & & 5.69 & & \\
\hline & & TPS & 5.67 & 0 & 0.1 & 5.67 & 0.4 & 0.2 \\
\hline & & MCNPX & 5.66 & & & 5.65 & & \\
\hline & \multirow{3}{*}{$\mathrm{Z}_{80}-\mathrm{Z}_{20}$} & Measurements & 0.25 & & & 0.27 & & \\
\hline & & TPS & 0.22 & -0.3 & -0.6 & 0.22 & -0.1 & -0.6 \\
\hline & & MCNPX & 0.28 & & & 0.28 & & \\
\hline
\end{tabular}

and should be performed following the previously established methodology (Farah et al., 2014). Further adjustments of some beamline elements and source characteristics could thus be required.

It should be noted that MC modeling of a beamline using the passive double-scattering technique is laborious, since a change in proton beam energy involves a change in beamline elements. Therefore, all various beamline elements need to be accurately modeled so as to perform calculations with any possible beamline configuration.

The comparison of MCNPX calculation results against TPS data also shows another limitation of this work. Indeed, in this simplistic water phantom configuration, the TPS slightly outperformed the simulations. Additional MC model improvements may hence be needed to reach a higher degree of accuracy and further be considered for a TPS commissioning, as suggested by Newhauser et al. (2005).

Finally, one should always bear in mind that MC calculations involve huge efforts to build an entire facility model, deep knowledge of simulation codes and non-negligible simulation time. These efforts can reasonably be invested in research-oriented facilities but limit the potential of this approach for daily treatment planning in proton therapy.

\section{Conclusion}

In this work, the previous MC model of the CPO's gantry room was upgraded, expanded and completed to consider new beamline configurations involving the use of two new RMWs, a new SS device and three new combinations of FS foils. Three realistic and clinically relevant beamline configurations were simulated, and both experimental measurements and TPS data were used for the validation process. Beamline elements were adapted with respect to the proton beam energies used of $162 \mathrm{MeV}, 192 \mathrm{MeV}$ and $219 \mathrm{MeV}$ at the beamline entrance.

A generally good agreement between the MCNPX calculations, experimental measurements and TPS data was found for PDD distributions and modulated lateral dose profiles, which did not differ by more than $2 \mathrm{~mm}$. This accuracy ensures the reliability of the methodology used for the source modeling, which can be reasonably applied to any proton beam energy 
Table 6. Comparison of MCNPX calculations against experimental measurements and TPS data in terms of field width at $90 \%$ ( $\left.\mathrm{Z}_{90}-\mathrm{Z}_{90}\right)$ and half maximum $\left(\mathrm{z}_{50}-\mathrm{Z}_{50}\right)$ and lateral penumbra $\left(\mathrm{z}_{80}-\mathrm{Z}_{20}\right)$ obtained at the end of the SOBP.

\begin{tabular}{|c|c|c|c|c|c|c|c|c|}
\hline \multicolumn{3}{|c|}{$\begin{array}{c}\text { Depth: } \\
\text { End of the SOBP }\end{array}$} & \multicolumn{3}{|c|}{ In-plane direction $(X)$} & \multicolumn{3}{|c|}{ Cross-plane direction (Y) } \\
\hline & & & $\begin{array}{c}\text { Comp. } \\
\text { values } \\
{[\mathrm{cm}]}\end{array}$ & $\begin{array}{c}\text { Meas. - } \\
\text { MCNPX } \\
{[\mathrm{mm}]}\end{array}$ & $\begin{array}{c}\text { TPS - } \\
\text { MCNPX } \\
{[\mathrm{mm}]}\end{array}$ & $\begin{array}{c}\text { Comp. } \\
\text { values } \\
{[\mathrm{cm}]}\end{array}$ & $\begin{array}{c}\text { Meas. - } \\
\text { MCNPX } \\
{[\mathrm{mm}]}\end{array}$ & $\begin{array}{c}\text { TPS - } \\
\text { MCNPX } \\
{[\mathrm{mm}]}\end{array}$ \\
\hline \multirow{9}{*}{$162 \mathrm{MeV}$ conf. } & \multirow{3}{*}{$\mathrm{Z}_{90}-\mathrm{Z}_{90}$} & Measurements & 4.93 & \multirow{3}{*}{1.2} & \multirow{3}{*}{0.6} & 4.94 & \multirow{3}{*}{1.2} & \multirow{3}{*}{0.5} \\
\hline & & TPS & 4.87 & & & 4.87 & & \\
\hline & & MCNPX & 4.81 & & & 4.82 & & \\
\hline & \multirow{3}{*}{$\mathrm{z}_{50}-\mathrm{Z}_{50}$} & Measurements & 5.75 & \multirow{3}{*}{-0.2} & \multirow{3}{*}{0.7} & 5.77 & \multirow{3}{*}{0} & \multirow{3}{*}{0.7} \\
\hline & & TPS & 5.84 & & & 5.84 & & \\
\hline & & MCNPX & 5.77 & & & 5.77 & & \\
\hline & \multirow{3}{*}{$\mathrm{Z}_{80}-\mathrm{Z}_{20}$} & Measurements & 0.52 & \multirow{3}{*}{-1.2} & \multirow{3}{*}{-0.8} & 0.55 & \multirow{3}{*}{-1.0} & \multirow{3}{*}{-0.9} \\
\hline & & TPS & 0.56 & & & 0.56 & & \\
\hline & & MCNPX & 0.64 & & & 0.65 & & \\
\hline \multirow{9}{*}{$192 \mathrm{MeV}$ conf. } & \multirow{3}{*}{$\mathrm{Z}_{90}-\mathrm{Z}_{90}$} & Measurements & 4.63 & \multirow{4}{*}{1.2} & \multirow{3}{*}{0.4} & 4.64 & \multirow{3}{*}{0.9} & \multirow{3}{*}{0} \\
\hline & & TPS & 4.55 & & & 4.55 & & \\
\hline & & MCNPX & 4.51 & & & 4.55 & & \\
\hline & \multirow{3}{*}{$\mathrm{Z}_{50}-\mathrm{Z}_{50}$} & Measurements & 5.92 & & \multirow{3}{*}{1.0} & 5.95 & \multirow{3}{*}{-0.4} & \multirow{3}{*}{0.3} \\
\hline & & TPS & 6.02 & \multirow[t]{2}{*}{0} & & 6.02 & & \\
\hline & & MCNPX & 5.92 & & & 5.99 & & \\
\hline & & Measurements & 0.84 & & & 0.86 & & \\
\hline & $\mathrm{Z}_{80}-\mathrm{Z}_{20}$ & TPS & 0.86 & -0.9 & -0.7 & 0.86 & -0.3 & -0.3 \\
\hline & & MCNPX & 0.93 & & & 0.89 & & \\
\hline & & Measurements & 4.48 & & & 4.39 & & \\
\hline & $\mathrm{Z}_{90}-\mathrm{Z}_{90}$ & TPS & 4.30 & 2.0 & 0.2 & 4.30 & 0.6 & -0.3 \\
\hline & & MCNPX & 4.28 & & & 4.33 & & \\
\hline & & Measurements & 6.02 & & & 6.03 & & \\
\hline $219 \mathrm{MeV}$ conf. & $\mathrm{z}_{50}-\mathrm{Z}_{50}$ & TPS & 6.12 & -0.4 & 0.6 & 6.12 & -0.1 & 0.8 \\
\hline & & MCNPX & 6.06 & & & 6.04 & & \\
\hline & & Measurements & 1.02 & & & 1.04 & & \\
\hline & $\mathrm{Z}_{80}-\mathrm{Z}_{20}$ & TPS & 1.07 & -1.5 & -1.0 & 1.07 & -1.6 & -1.3 \\
\hline & & MCNPX & 1.17 & & & 1.20 & & \\
\hline
\end{tabular}

modeled for the CPO's gantry beamline, while the reproduction of the beamline components tested was also validated. However, additional testing of the MC code in the simulation of secondary neutrons is still needed. Nonetheless, the accuracy achieved is sufficient to carry out investigations which aim at assessing secondary neutron doses to healthy tissues in order to improve the radiation protection of the patient. Current work thus involves the use of these new beamline configurations to study the variability of neutron doses with proton beam energy and also some other irradiation parameters.

\section{References}

Athar B.S., Paganetti H. (2011) Comparison of second cancer risk due to out-of-field doses from 6-MV IMRT and proton therapy based on 6 pediatric patient treatment plans, Radiother. Oncol. 98, 87-92.

Bertini H.W. (1963) Low-energy intranuclear cascade calculation, Phys. Rev. 131, 1801-1821.

Chadwick M.B. (1998) Neutron, proton, and photonuclear crosssections for radiation therapy and radiation protection, Radiat. Environ. Biophys. 37, 235-242.
Chadwick M.B., Young P.G., Chiba S., Frankle S.C., Hale G.M., Hughes H.G., Koning A.J., Little R.C., MacFarlane R.E., Prael R.E., Waters L.S. (1999) Cross-Section evaluations to $150 \mathrm{MeV}$ for Accelerator-Driven Systems and implementation in MCNPX, Nucl. Sci. Eng. 131, 293-328.

Dresner L. (1962) EVAP: a fortran program for calculating the evaporation of various particles from excited compound nuclei, Oak Ridge National Laboratory report ORNL-TM-196.

Faddegon B.A., Shin J., Castenada C.M., Ramos-Méndez J., Daftari K. (2015) Experimental depth dose curves of a $67.5 \mathrm{MeV}$ proton beam for benchmarking and validation of Monte Carlo simulation, Med. Phys. 42, 4199-4210.

Farah J., Martinetti F., Sayah R., Lacoste V., Donadille L., Trompier F., Nauraye C., De Marzi L., Vabre I., Delacroix S., Hérault J., Clairand I. (2014) Monte Carlo modeling of proton therapy installations: a global experimental method to validate secondary neutron dose calculations, Phys. Med. Biol. 59, 2747-2765.

Fontenot J., Taddei P., Zheng Y., Mirkovic D., Jordan T., Newhauser W. (2008) Equivalent dose and effective dose from stray radiation during passively scattered proton radiotherapy for prostate cancer, Phys. Med. Biol. 53, 1677-1688. 
Gottschalk B., Koehler A.M., Schneider R.J., Sisterson J.M., Wagner M.S. (1993) Multiple Coulomb scattering of $160 \mathrm{MeV}$ protons, Nucl. Instrum. Methods Phys. Res. B 74, 467-490.

Hall E.J. (2006) Intensity-modulated radiation therapy, protons, and the risk of second cancers, Int. J. Radiat. Oncol. Biol. Phys. 65, $1-7$.

Hérault J., Iborra N., Serrano B., Chauvel P. (2005) Monte Carlo simulation of a proton therapy platform devoted to ocular melanoma, Med. Phys. 32, 910-919.

Hérault J., Iborra N., Serrano B., Chauvel P. (2007) Spread-out Bragg peak and monitor units calculation with the Monte Carlo Code MCNPX, Med. Phys. 34, 680-688.

Hong L., Goitein M., Bucciolini M., Comiskey R., Gottschalk B., Rosenthal S., Serago C., Urie M. (1996) A pencil beam algorithm for proton dose calculations, Phys. Med. Biol. 41, 1305-1330.

IBA (2007) Ionization chambers and diode detectors, Detectors for relative and absolute dosimetry, IBA dosimetry.

Mesoloras G., Sandison G.A., Stewart R.D., Farr J.B., Hsi W.C. (2006) Neutron scattered dose equivalent to a fetus from proton radiotherapy of the mother, Med. Phys. 33, 2479-2490.

Miralbell R., Lomax A., Cella L., Schneider U. (2002) Potential reduction of the incidence of radiation-induced second cancers by using proton beams in the treatment of pediatric tumors, Int. J. Radiat. Oncol. Biol. Phys. 54, 824-829.

Newhauser W., Koch N., Hummel S., Ziegler M., Titt U. (2005) Monte Carlo simulations of a nozzle for the treatment of ocular tumours with high-energy proton beams, Phys. Med. Biol. 50, 5229-5249.

Newhauser W.D., Fontenot J.D., Mahajan A., Kornguth D., Stovall M., Zheng Y., Taddei P.J., Mirkovic D., Mohan R., Cox J.D., Woo S. (2009) The risk of developing a second cancer after receiving craniospinal proton irradiation, Phys. Med. Biol. 54, 2277-2291.
Pelowitz D.B. (2008) MCNPX User's Manual Version 260, LA-CP07-1473.

Polf J.C., Harvey M.C., Titt U., Newhauser W.D., Smith A.R. (2007) Initial beam size study for passive scatter proton therapy I. Monte Carlo verification, Med. Phys. 34, 4213-4218.

Rossi B., Greisen K. (1941) Cosmic-ray theory, Rev. Mod. Phys. 13, 240-309.

Sayah R., Donadille L., Aubé A., Hérault J., Delacroix S., De Marzi L., Stichelbaut F., Clairand I. (2013) Monte Carlo simulation of a proton therapy beam line for intracranial treatments, Radioprotection 48, 317-339.

Sayah R., Farah J., Donadille L., Hérault J., Delacroix S., De Marzi L., De Oliveira A., Vabre I., Stichelbaut F., Lee C., Bolch W.E., Clairand I. (2014) Secondary neutron doses received by paediatric patients during intracranial proton therapy treatments, $J$. Radiol. Prot. 34, 279-296.

Shin D., Yoon M., Kwak J., Shin J., Lee S.B., Park S.Y., Park S., Kim D.Y., Cho K.H. (2009) Secondary neutron doses for several beam configurations for proton therapy, Int. J. Radiat. Oncol. Biol. Phys. 74, 260-265.

Szymanowski H., Mazal A., Nauraye C., Biensan S., Ferrand R., Murillo M.C., Caneva S., Gaboriaud G., Rosenwald J.C. (2001) Experimental determination and verification of the parameters used in a proton pencil beam algorithm, Med. Phys. 28, 975-987.

Vavilov P.V. (1957) Ionization losses of high-energy heavy particles, Sov. Phys. JETP 5, 749-751.

Zacharatou Jarlskog C., Lee C., Bolch W.E., Xu X.G., Paganetti H. (2008) Assessment of organ specific neutron equivalent doses in proton therapy using computational whole-body age-dependent voxel phantoms, Phys. Med. Biol. 53, 693-717.

Cite this article as: A. Bonfrate, J. Farah, L. De Marzi, S. Delacroix, E. Constant, J. Hérault, I. Clairand. Benchmarking Monte Carlo simulations against experimental data in clinically relevant passive scattering proton therapy beamline configurations. Radioprotection 51(2), 113-122 (2016). 